\title{
RNA interference-mediated downregulation of Beclin1 attenuates cerebral ischemic injury in rats
}

\author{
Yong-qiu ZHENG, Jian-xun LIU*, Xin-zhi LI, Li XU, Yong-gang XU \\ Research Center, Xiyuan Hospital, China Academy of Chinese Medical Sciences, Beijing 100091, China
}

\begin{abstract}
Aim: To test the role of the Beclin 1-dependent autophagy pathway in brain damage during cerebral ischemia.
Methods: Focal cerebral ischemia was established in rats using a middle cerebral artery occlusion (MCAO) model. A lentiviral vectorassociated RNA interference (RNAi) system was stereotaxically injected into the ipsilateral lateral ventricle to reduce Beclin1 expression. We measured the ipsilateral infarct volume, autophagosome formation, neurogenesis and apoptosis, all of which could be modulated by Beclin1 RNAi.

Results: On the 14th day after MCAO, Beclin1 downregulation by RNAi increased the population of neural progenitor cells $\left(\mathrm{BrdU}^{+}\right.$-DCX ${ }^{+}$, newborn immature cells (BrdU $\left.-\mathrm{Tuj}-1^{+}\right)$and mature neurons $\left(\mathrm{BrdU}^{+}-\mathrm{MAP}-2^{+}\right)$, and reduced the apoptosis of immature neurons (caspase$3^{+}-\mathrm{DCX}^{+}$and caspase- $\left.3^{+}-\mathrm{Tuj}-1^{+}\right)$surrounding the ischemic core of the ipsilateral hemisphere. Furthermore, RNAi-mediated downregulation of Beclin1 decreased infarct volume and inhibited histological injury and neurological deficits.
\end{abstract}

Conclusion: RNAi-mediated downregulation of Beclin1 improves outcomes after transient MCAO.

Keywords: autophagy; beclin1; apoptosis; stroke; RNA interference

Acta Pharmacologica Sinica (2009) 30: 919-927; doi: 10.1038/aps.2009.79

\section{Introduction}

Apoptosis and necrosis are important modes of neuronal death induced by cerebral ischemia, but in recent years a third method of death, autophagy, has been studied ${ }^{[1-3]}$. Autophagy, which involves the formation of autophagosomes and autophagolysosomes, has been studied extensively as a caspase-independent cell death pathway and has been associated with ischemia and reperfusion in the heart and brain ${ }^{[4-7]}$. Autophagy appears to occur after hypoxia-ischemia as an important mechanism of neuronal self-destruction, as well as after transient global ischemia and adult focal ischemia ${ }^{[6-10]}$. In addition, autophagy has also been recognized as a cell survival mechanism. It may protect neurons by eliminating damaged structures and delivering them to lysosomes for degradation $^{[11,12]}$. Hence, whether autophagy is a friend or foe during cerebral ischemia/reperfusion remains to be seen. We therefore used a rat middle cerebral artery occlusion (MCAO) model to induce transient focal brain ischemia in the present study.

Beclin1, the homologue of the yeast autophagy gene 6 (Atg6), was first dissociated from the inhibitory Beclin1-

\footnotetext{
* To whom correspondence should be addressed.

E-mail Liujx0324@sina.com

Received 2009-01-12 Accepted 2009-04-28
}

Bcl-2 complex and was suggested as a potential link between autophagy and apoptosis ${ }^{[13]}$. Beclin1-deficient mice show a pronounced loss of autophagic vacuole formation ${ }^{[14-16]}$. In mammals, Beclin1 downregulation by RNA interference (RNAi) blocked cell death induced by autophagy ${ }^{[17,18]}$. Furthermore, induction of autophagy and cardiac injury during the reperfusion phase were significantly attenuated in Beclin $1^{-} /^{-}$mice ${ }^{[5]}$. As a rapamycin-independent autophagy mechanism ${ }^{[12]}$, the role of the Beclin1-induced autophagy in brain ischemia needs more elucidation. Therefore, in the present study, lentiviral vectors encoding Beclin1 short hairpin RNA (LV-shBeclin1) were stereotaxically delivered into the ipsilateral lateral ventricle to inhibit ischemia-induced Beclin1 expression.

As previously reported, an increase in neurogenesis following ischemia has been considered to be necessary for functional recovery after stroke ${ }^{[19,20]}$. Beclin1-dependent autophagy has been associated with neurogenesis in several models ${ }^{[21,22]}$. These findings supporting the existence of a complex interplay between autophagy, cell proliferation and cell death during neural development ${ }^{[22]}$. However, it remains unknown whether autophagy has any influence on ischemia-induced neurogenesis. The present study addresses the role of Beclin1dependent autophagy during the period of neurogenesis after transient MCAO. 
Here, we demonstrate that RNAi-mediated downregulation of Beclin1 was protective following transient $\mathrm{MCAO}$ in vivo. Further, the present study is the first, to our knowledge, to examine the potential link between Beclin1-dependent autophagy, apoptosis, and neurogenesis in response to cerebral ischemia/ reperfusion.

\section{Materials and methods Animals}

Adult male Sprague-Dawley rats (250-270 g), provided by the Department of Animal, Health Science Center of Peking University, were housed in a laboratory animal room and maintained at $25 \pm 1{ }^{\circ} \mathrm{C}$ with $65 \% \pm 5 \%$ humidity on a 12-h light/ dark cycle (lights on from 07:30 to 19:30) for at least 1 week before the start of the experiments. Animals were given food and water ad libitum. All experimental protocols described in this study were approved by the Ethics Review Committee for Animal Experimentation of Xiyuan Hospital, China Academy of Chinese Medical Sciences.

\section{Lentiviral vectors and shRNA injection}

The design of the shRNA followed published guidelines $^{[23]}$. Lentiviral vectors pGCL-LTR-RRE-U6 shRNA-CMV Luciferase-WRE-LTR (LV-shBeclin1) were generated. The shBeclin1 sense oligonucleotide sequence 5'-GGATCCCAACTCACAGCTCCATTACTTATTCAAGAGATAAGTAATGGAGCTGTGAGTTTTTTTGGATAGCTT-3' and the shBeclin1 antisense oligonucleotide sequence 5'-AAGCTATCCAAAAAAACTCACAGCTCCATTACTTATCTCTTGAATAAGTAATGGAGCTGTGAGTTGGGATCC-3' were annealed and cloned into a pGC/U6 plasmid. One unrelated sequence (TTCTCCGAACGTGTCACGT) that shows no significant homology to any known rat gene (analyzed using a BLAST search) was used as an LV-control. Seven days before MCAO, $1.5 \mu \mathrm{L}$ of concentrated viral solution was stereotaxically delivered into the ipsilateral lateral ventricle (coordinates from bregma: AP-0.8 mm, ML $1.4 \mathrm{~mm}, \mathrm{DV}-3.6 \mathrm{~mm}$ from the pial surface).

\section{Transient MCAO}

Rats with physiological variables within normal ranges were subjected to transient focal cerebral ischemia induced by right MCAO as previously described ${ }^{[24]}$. In brief, rats were anesthetized with $3.5 \%$ chloral hydrate $(350 \mathrm{mg} / \mathrm{kg}$, ip) and were placed in a supine position. The body temperature of the rats was maintained at $37 \pm 0.5{ }^{\circ} \mathrm{C}$ during the surgical procedure. The right common carotid artery (CCA), internal carotid artery (ICA), and external carotid artery (ECA) were surgically exposed. The CCA was ligated distally and the ECA was ligated proximally to the bifurcation of the ICA and the ECA. A 3-0 silk suture was tied loosely around the ICA, and a microvascular clip was placed distally across the ICA. A 4-0 nylon monofilament suture with a rounded tip was inserted into the ICA through the CCA and gently advanced for a distance of $20 \mathrm{~mm}$ from the common carotid artery bifurcation to block the origin of the middle cerebral artery (MCA). The suture around the ICA was tightened, and the microvasculature clip was removed. Mean arterial blood pressure, heart rate, and arterial blood gases were analyzed during the surgical procedure. After $60 \mathrm{~min}$ of $\mathrm{MCAO}$, the suture was pulled back until the tip reached the suture around the ICA to restore blood flow (reperfusion). Regional cerebral blood flow (rCBF) was measured during the focal ischemia surgery. Laser-Doppler flowmetry (Periflux, Perimed) was used to monitor rCBF. Recordings were made bilaterally from each animal for at least $10 \mathrm{~min}$ before, during, and within $30 \mathrm{~min}$ after reperfusion. After the wound had been closed, the animals were allowed to recover from anesthesia before they were returned to their home cages.

\section{Luciferase assay}

Luciferase activity was quantified by a luciferase assay system (Promega, Madison, WI) according to the manufacturer's protocol. The results were reported as luciferase activity/total protein.

\section{Tissue preparation}

Rats were sacrificed $24 \mathrm{~h}$ and 14 days after MCAO with an overdose of $3.5 \%$ chloral hydrate and were transcardially perfused with $0.9 \%$ saline solution followed by $4 \%$ ice-cold phosphate-buffered paraformaldehyde (PFA). Brains were removed, postfixed overnight, and equilibrated in phosphatebuffered $30 \%$ sucrose. Coronal sections at 1.0 to $0.2 \mathrm{~mm}$ from bregma were used for immunohistochemical staining and were cut on a freezing microtome (Leica CM3000, Germany) at a thickness of $25 \mu \mathrm{m}$.

\section{Immunohistochemistry and immunofluorescence staining}

Frozen sections were double-stained for immunofluorescence and cell phenotypes were identified by colocalization of phenotypic markers with BrdU, as previously described, using these primary antibodies (and their concentrations): Goat polyclonal anti-doublecortin (DCX) to label neural progenitor cells (1:100; Santa Cruz); rabbit polyclonal anti-microtubuleassociated protein-2 (MAP-2) to label mature neurons (1:50; Cell Signaling); mouse monoclonal anti- $\beta$ III tubulin (Tuj-1) to label immature neurons (1:100; Chemicon, Temecula), and rabbit polyclonal anti-caspase-3 to label apoptosis of neurons (1:100; cell signaling). Secondary antibodies: anti-mouse, rabbit and goat IgG-fluorescein isothiocyanate (FITC) and IgGCy3 (1:200; Chemicon). Confocal images were taken with a Zeiss LSM-510 microscope.

\section{Transmission electron microscopy (TEM)}

TEM was used to evaluate the ultrastructural changes. Cerebral fragments were fixed with $2.5 \%$ glutaraldehyde solution overnight at $4{ }^{\circ} \mathrm{C}$ and were washed with PBS after being fixed with $1 \%$ osmic acid for $2 \mathrm{~h}$. The samples were embedded in an Epon/Araldite mixture and stained with uranyl acetate and lead citrate, and then were observed under a 1230 type transmission electron microscope (Electron Co, Japan) and photographed. 


\section{Western blotting}

Rats were sacrificed at 4,10 , and $24 \mathrm{~h}$ after reperfusion with an overdose of $3.5 \%$ chloral hydrate. Rat cerebral homogenates were collected and centrifuged for $4 \mathrm{~min}$ at $3000 \times \mathrm{g}$ and the resulting supernatants spun at $20000 \times g$ for $30 \mathrm{~min}$. Supernatants were diluted into $0.5 \mathrm{mg}$ protein/ $\mathrm{mL}$ for the measurement of Beclin1 and LC3. Protein content was determined with BSA as a standard according to the Bradford method ${ }^{[25]}$. Protein samples (20 $\mathrm{\mu g} /$ lane) were separated by sodium dodecyl sulfate-polyacrylamide gel electrophoresis and transferred to PVDF membranes (Millipore, Billerica, MA) through electroblotting. Blots were stained with rabbit polyclonal anti-autophagy APG8a (MAP1LC3A) antibody (Abgent, San Diego, CA, USA) and rabbit polyclonal anti-Beclin1 antibody (Cell Signaling). Then the blots were developed by enhanced chemiluminescence using SuperSignal west femto maximum sensitivity substrate (Pierce, Rockford, IL) and Kodak X-AR film. The Bio-Rad Quantity One ${ }^{\circledR} 1-D$ Analysis Software system, version 4.4 , was used to calculate the numerical value of every blot, and mean densitometric $\times$ areal values were depicted as bar graphs. All the experiments reported in this study were performed three times and the results were reproducible.

\section{Measurement of infarct volume}

At 14 days after reperfusion, the rats were deeply anesthetized with $3.5 \%$ chloral hydrate and then decapitated, after which the brains were rapidly removed. Coronal sections $(n=6$ for each group) were cut into 2 -mm slices and stained with standard 2\% 2,3,5-triphenyltetrazolium chloride (TTC, SigmaAldrich) for $10 \mathrm{~min}$ at $37^{\circ} \mathrm{C}$ followed by overnight immersion in $4 \%$ formalin. Infarct volume, expressed as a percentage of whole-brain volume, was measured by an image processing and analysis system (1.25×objective, Q570IW, Leica, Germany) and was calculated by integration of the infarct area in each brain section along the rostral-caudal axis ${ }^{[26]}$.

\section{Neurological deficit scores}

Neurological deficit scores were determined at 24 h, 7 days and 14 days after MCAO as previously described ${ }^{[27]}$ : no neurological deficit (value=0); left forelimb flexion when suspended by the tail or failure to extend right forepaw fully (value=1); left shoulder adduction when suspended by the tail (value $=2$ ); reduced resistance to lateral push toward the left side (value=3); spontaneous movement in all directions with circling to the left exhibited only if pulled by tail (value=4); circle or walk spontaneously only to the left (value=5); walk only when stimulated (value $=6$ ); no response to stimulation (value=7); and stroke-related death (value=8).

\section{Hematoxylin and eosin (H\&E) staining}

The sections were stained using H\&E according to the standard procedures and observed under an Olympus BX51 light microscope. Under a light microscope at 400× magnification, 5 nonoverlapping fields in each of the selected regions of inter- est (ROIs) were examined to determine the intact neurons and injured neurons.

\section{Data quantification and statistical analysis}

Regions of interest (ROIs) were defined as a zone with $250 \mu \mathrm{m}$ width and $800 \mu \mathrm{m}$ length in the SVZ, and as a box with $700 \mu \mathrm{m}$ width and length in the peri-infarct striatum and cortex (Figure 1A). For immunofluorescence counting, four sections were obtained and the results were expressed as the average number per rat. All data were expressed as mean \pm SD. Statistical analysis was performed using SPSS, version 11.0. ANOVA or a nonparametric test was used for statistical analysis. $P<0.05$ was considered statistically significant.

\section{Results}

\section{Autophagy activation surrounding the ischemic core}

Beclin1 has been identified as a mammalian autophagy gene $^{[28]}$. In the present study, cellular protein extracts from the ipsilateral cortex, striatum and dentate gyrus (DG) surrounding the ischemic core were assessed by Western blot analysis. Here, the expression of Beclin1 was significantly enhanced in all regions as early as $4 \mathrm{~h}$ after ischemia (Figure 1B-1D). LC3-II is correlated with the extent of autophagosome formation and LC3-II/LC3-I provides a important molecular marker for detection of autophagic activity ${ }^{[14,29]}$. The present study indicated that the LC3-II/LC3-I ratio was significantly increased in the ipsilateral striatum and hippocampus at 4, 10, and $24 \mathrm{~h}$ after reperfusion and in the ipsilateral cortex at $24 \mathrm{~h}$ after reperfusion, as compared to sham operated animals (Figure 1B-1D). The structures of autophagosomes, bounded by two or more membranes and containing cytoplasmic ground substance or fragments of cellular organelles, were frequently observed in ipsilateral neurons (Figure 2). The results demonstrate that autophagy is activated by transient MCAO.

\section{Beclin1 expression in ipsilateral hemisphere treated with LV- shBeclin1}

In order to test whether I/R-induced autophagic injury was dependent on Beclin1 expression, we depleted endogenous Beclin1 levels by stereotaxic injection of LV-shBeclin1 7d before ischemia. Luciferase activity was quantified as the reporter in the present study. At day 3 (preoperative day 4), animals treated with lentiviral vectors containing the luciferase sequence demonstrated positive luciferase expression, which peaked at day 14 (Figure 3C). We showed that LV-shBeclin1 led to a reduction of Beclin1 protein compared with LV-control in targeted regions such as the ipsilateral cortex, striatum and DG in I/ $R$ rats (Figure 3A, 3B, 3D). There were no significant differences in the studied parameters between LV-control and LV-shBeclin1 groups (Table 1). In both groups, CBF was reduced to approximately $25 \%$ of baseline during MCAO and recovered during the reperfusion phase, reaching $57 \%$ and $62 \%$ of baseline in the LV-control and LV-shBeclin 1 groups, respectively. There were no significant differences in the CBF between the LV-control and LV-shBeclin1 groups (Figure 3E). 
A

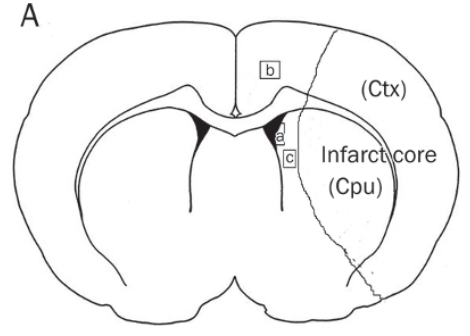

B

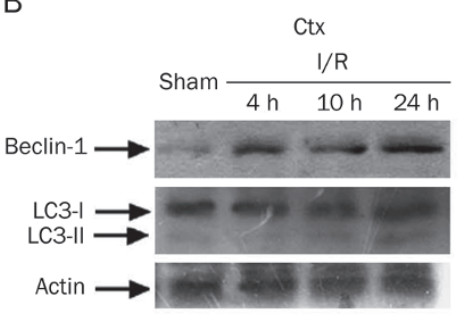

Ipsilateral hemisphere

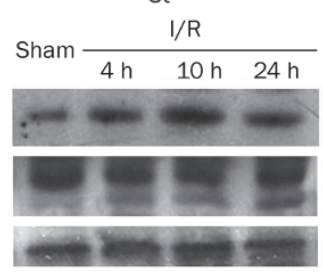

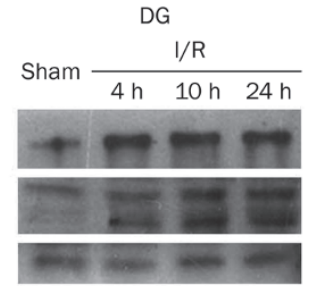

$\mathrm{C}$
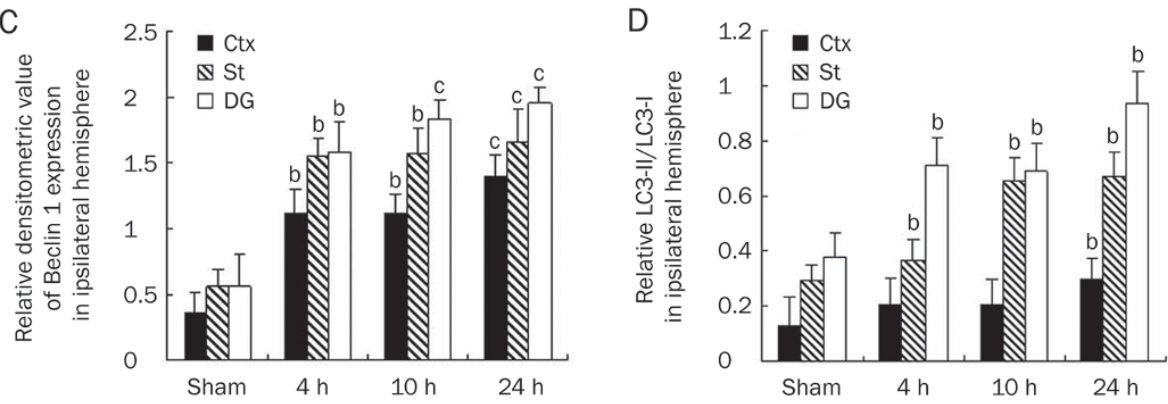

Figure 1. Beclin1 expression and LC3-II/LC3-I in ipsilateral hemisphere. (A) Diagram of the brain section. ROls were defined in the ipsilateral SVZ (a), Ctx (b), and St (c). (B) Western blot analysis of Beclin1, LC3-II, and LC3-I expression in ipsilateral cortex, striatum and DG at different times after reperfusion, as described in the text. Results were obtained from three independent experiments; only one representative experiment is shown. (C and D) Bar graphs show quantitative evaluation of Beclin1 expression and LC3-II/LC3-I in the ipsilateral hemisphere. $n=3$. Mean \pm SD. A nonparametric test was used for statistical analysis. ${ }^{b} P<0.05,{ }^{c} P<0.01$ compared with sham group. Ctx, Cortex. St, Striatum. DG, dentate gyrus.

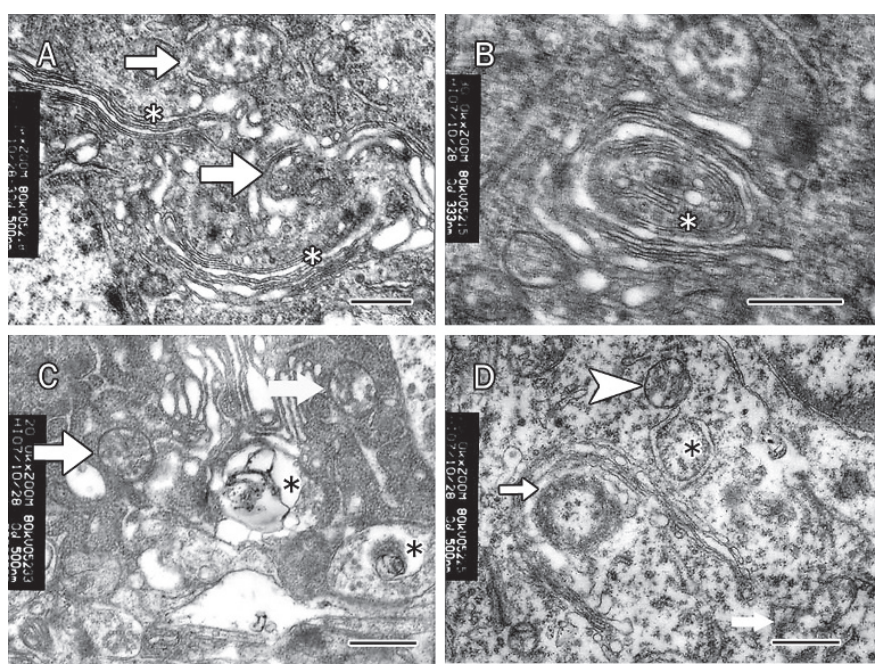

Figure 2. Ultrastructural morphology of several autophagic profiles. (A) An early stage of autophagic vacuole formation, identified as membranes from the rough endoplasmic reticulum containing cytosolic components (white asterisks) in neurons from the $D G$ at $10 \mathrm{~h}$ after reperfusion. This process leads to the formation of the autophagosomes, which are characterized by double membrane vesicles (white arrows). (B) An autophagosome with several double membranes in a neuron of the DG at $24 \mathrm{~h}$ after reperfusion (white asterisk). (C) Autophagosomes, adjacent to lysosomes (white arrows), contain some vacuoles bounded by a small inner vesicle enclosing residues of the lytic organelles (black asterisks) (36 $\mathrm{h}$ after reperfusion). (D) Autophagic vacuole with double membrane vesicles (black asterisk) adjacent to lysosomes (white arrowhead). Autophagolysosomes contain residues of membranes and lytic organelles (white arrows) (48 $\mathrm{h}$ after reperfusion). Scale bar: $500 \mathrm{~nm}$.

\section{Apoptosis of immature neurons surrounding the ischemic hemisphere}

Double labeling of active caspase-3 (fragment of p17) and DCX/Tuj-1 $\left(\mathrm{Casp}^{+}-\mathrm{DCX}^{+} / \mathrm{Casp}^{+}-\mathrm{Tuj}-1^{+}\right)$was performed in the present study. Activated caspase-3 immunostaining appeared in Tuj- $1^{+}$and $\mathrm{DCX}^{+}$cells in the ipsilateral striatum at 14 days of reperfusion. The Tuj- $1^{+}-\mathrm{Casp}-3^{+}$and $\mathrm{DCX} \mathrm{X}^{+}$-Casp $-3^{+}$cells were decreased by treatment with LV-shBeclin1 (Figure 4). The results indicated that downregulation of Beclin1 by RNAi reduced the apoptosis of immature neurons in response to reperfusion.

\section{Infarct volume}

Transient focal ischemia for 60 min caused infarction in the striatum and frontoparietal cortex on day 14 after reperfusion. Examination of serial brain sections showed that stereotaxic injection of LV-shBeclin1 into the ipsilateral lateral ventricle significantly decreased infarct volume compared with LV-control treatment 14 days after focal ischemia/reperfusion (Figure 5).

\section{Histological outcomes and neurological deficits}

In I/R rats treated with LV-control, most neurons were markedly shrunken, and some had pyknotic nuclei and eosinophilic cytoplasm near the boundary of the ischemic core. In the I/ R+LV-shBeclin1 group, many neurons were slightly shrunken and surrounded by swollen cellular processes (Figure 6A-6D). Neurological deficit scoring was performed from $24 \mathrm{~h}$ to 14 days after reperfusion. Neurological deficit scores 
A
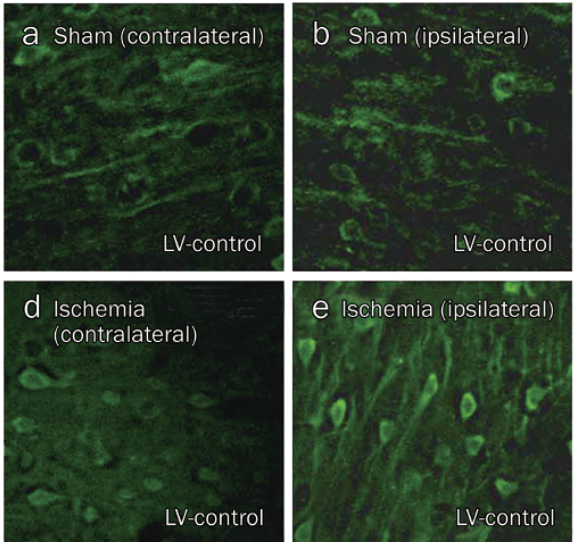

e Ischemia (ipsilateral) e
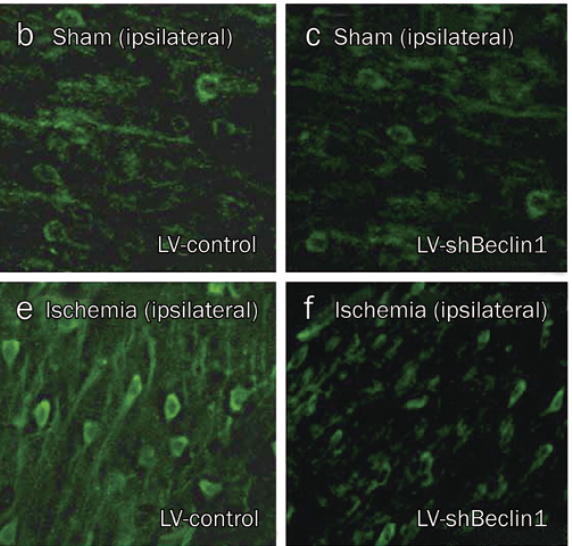

c

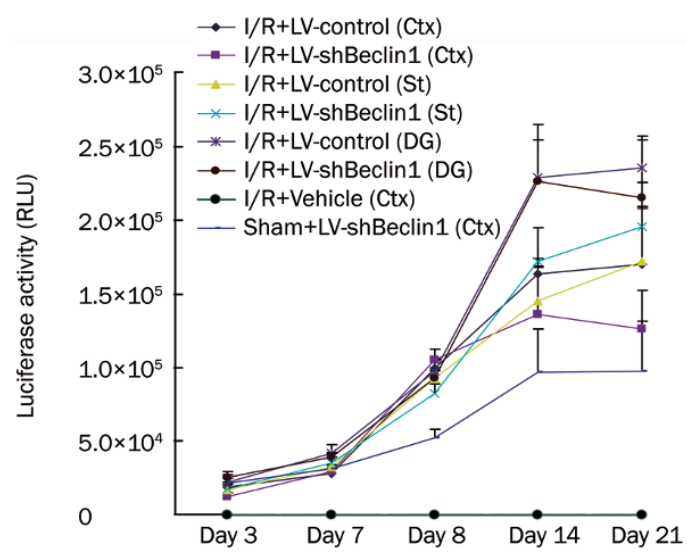

$\mathrm{E}$

CBF during $M C A O$ and after reperfusion
B
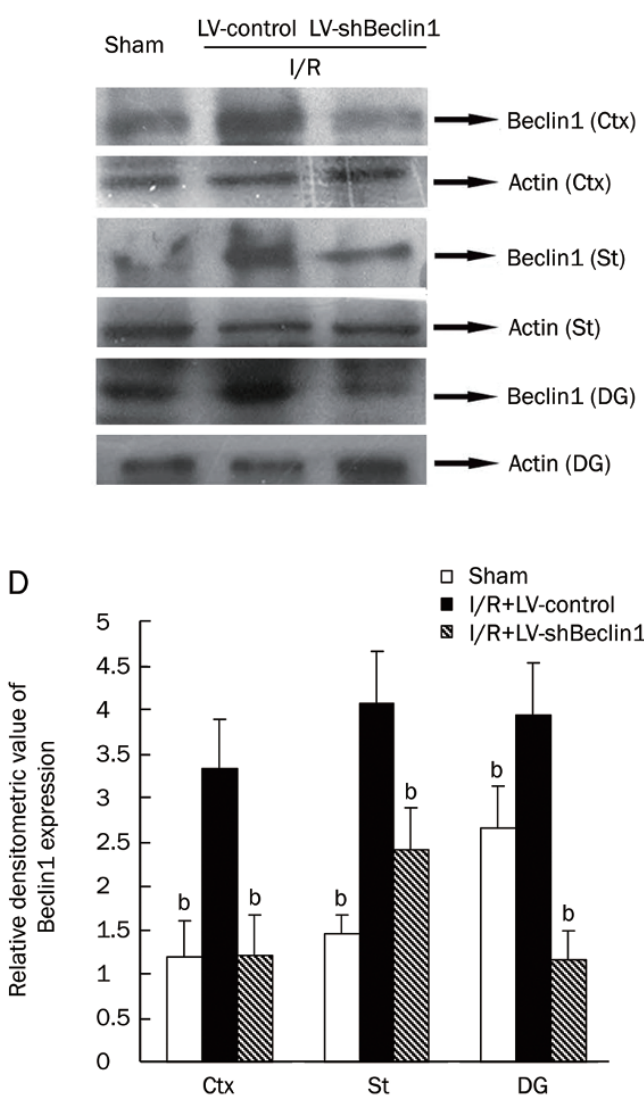

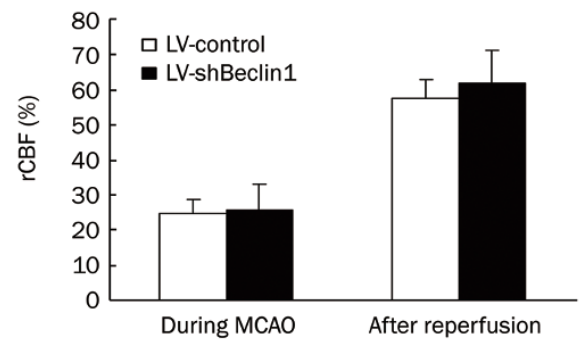

Figure 3. Beclin1 expression in ipsilateral hemisphere following stereotaxic injection of LV-shBeclin1 or LV-control. (A) Staining of Beclin1 (green) at 24 $\mathrm{h}$ post-ischemia indicates that Beclin1 expression in the ipsilateral hemisphere is inhibited by stereotaxic injection of LV-shBeclin1. (B) Western blot analysis of Beclin1 expression in the ipsilateral cortex, striatum and DG at $24 \mathrm{~h}$ after reperfusion. (C) Quantitative analysis of luciferase activity. $n=6$. Mean \pm SD. (D) Bar graphs show quantitative evaluation of Beclin1 expression. Results were obtained from three independent experiments; only one representative experiment is shown, $n=3$. Mean \pm SD. A nonparametric test was used for statistical analysis. ${ }^{b} P<0.05$ compared with $\mathrm{I} / \mathrm{R}+\mathrm{LV}-\mathrm{control}$ group. (E) CBF during MCAO and after reperfusion. $n=5$. Mean $\pm S D$. Ctx, Cortex. St, striatum. DG, dentate gyrus. I/R, Ischemia/Reperfusion.

decreased in the I/R+LV-shBeclin1 group and significant differences were found at $24 \mathrm{~h}$ and 14 days after reperfusion compared with the I/R+LV-control group (Figure $6 \mathrm{E}$ ). These findings indicate that LV-shBeclin1 protects against ischemic brain injury.

Accumulation, migration and maturity of newborn neurons

We next investigated whether reduction of Beclin1 expression by RNAi participated in neurogenesis in the ischemic brain. Double staining of BrdU and DCX (BrdU'-DCX ${ }^{+}$) was used to detect the proliferating neural progenitor cells. Increased number of $\mathrm{BrdU}^{+}-\mathrm{DCX}^{+}$cells was observed in the subventricular zone (SVZ) 14 days post-ischemia. Beclin1 RNAi increased $\mathrm{BrdU}^{+}-\mathrm{DCX}^{+}$cells in the ipsilateral SVZ of the MCAO group (Figure 7A-7B). To determine whether Beclin1 RNAi influenced differentiation of neural progenitor cells to neuronal 
Table 1. Physiological parameters in rats subjected to transient MCAO. $n=5$. Mean \pm SD.

\begin{tabular}{|c|c|c|c|c|c|}
\hline Group & & $\mathrm{MABP}(\mathrm{mmHg})$ & $\mathrm{pH}$ & $p_{\mathrm{CO}_{z}}(\mathrm{mmHg})$ & $p_{\mathrm{O}_{2}}(\mathrm{mmHg})$ \\
\hline Before MCAO & LV-control & $82 \pm 6$ & $7.35 \pm 0.01$ & $58 \pm 6$ & $67 \pm 2$ \\
\hline \multirow[t]{2}{*}{ Ischemia (30 min) } & LV-control & $83 \pm 3$ & $7.35 \pm 0.03$ & $49 \pm 2$ & $70 \pm 5$ \\
\hline & LV-shBeclin1 & $85 \pm 5$ & $7.35 \pm 0.02$ & $48 \pm 5$ & $73 \pm 7$ \\
\hline Reperfusion (30 min) & LV-control & $80 \pm 6$ & $7.35 \pm 0.02$ & $45 \pm 4$ & $79 \pm 6$ \\
\hline
\end{tabular}
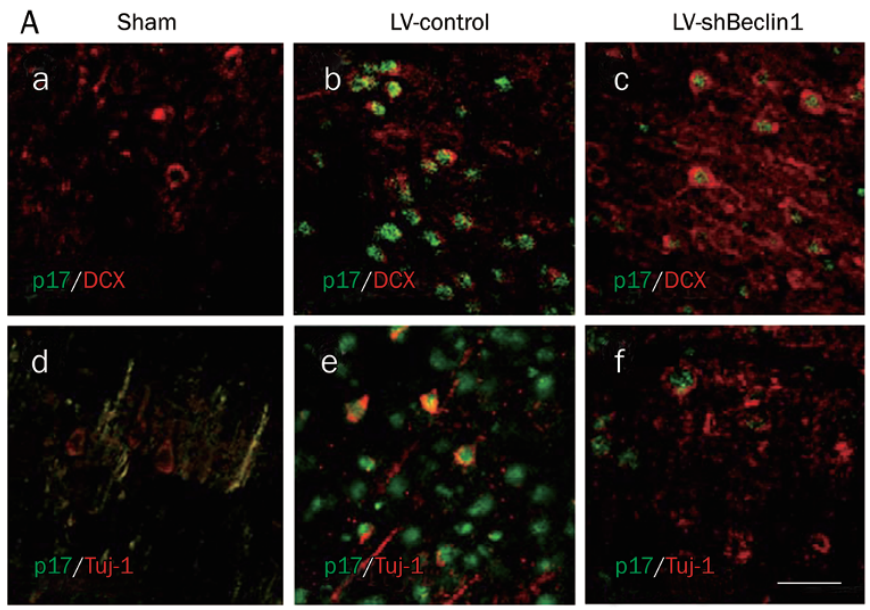

B
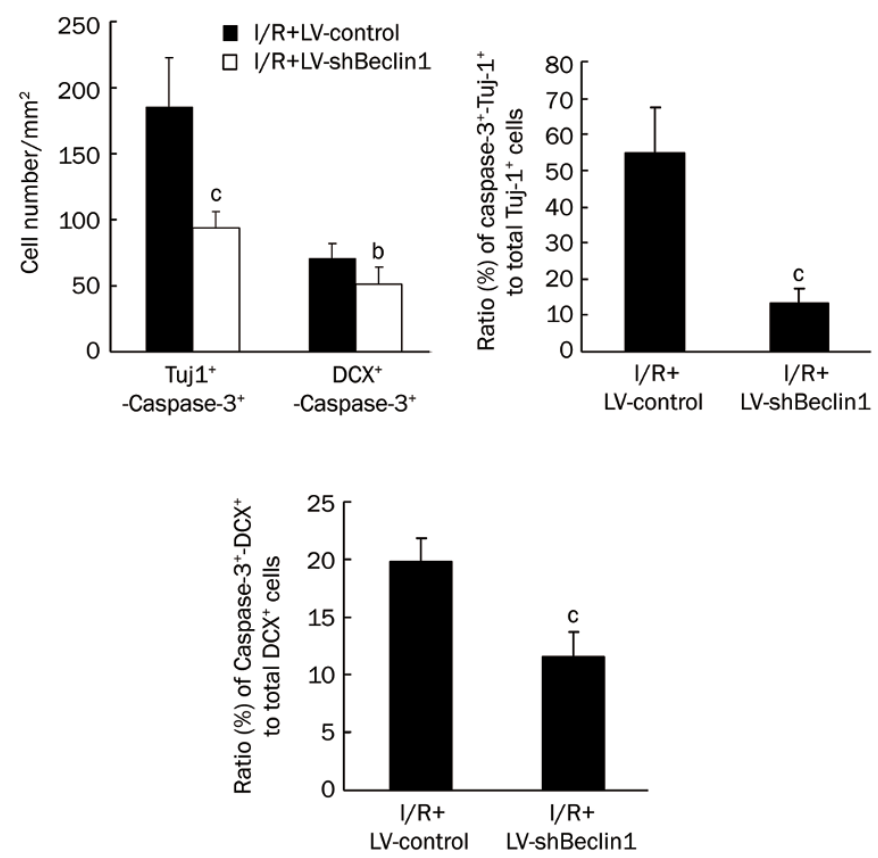

Figure 4. Apoptosis of new neurons. (A) The merged confocal images confirmed Tuj-1 (red), DCX (red) and caspase-3 (green) expressing cells in the ipsilateral striatum from animals treated with LV-shBeclin1 or LVcontrol 14 days after MCAO. (B) Quantitative data of each cell. An ANOVA test was used for statistical analysis. ${ }^{\mathrm{b}} P<0.05,{ }^{\mathrm{C}} P<0.01$ vs LV-control group. $n=6$. Data are mean \pm SD. Scale bar $=10 \mu \mathrm{m}$.
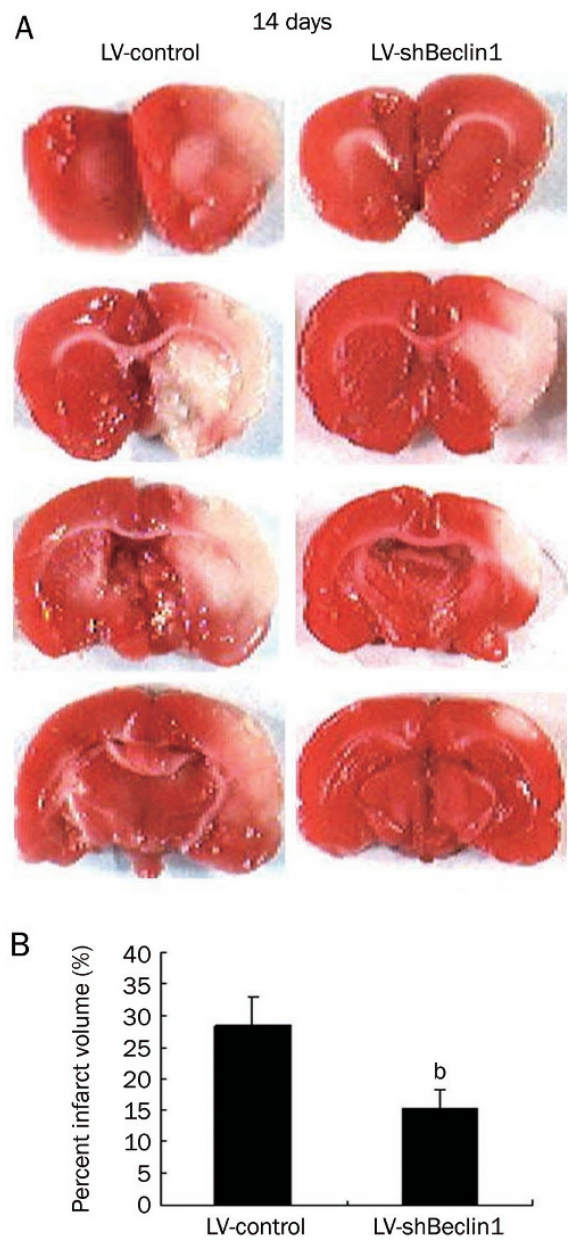

Figure 5. Infarct volume. (A) Representative TTC staining of the cerebral infarct in comparable sections of rat brain 14 days after ischemia. (B) Quantification of infarct volume. An ANOVA test was used for statistical analysis. ${ }^{\mathrm{b}} P<0.05$ vs LV-control group. $n=6$. Mean \pm SD.

lineage, we used BrdU ${ }^{+}-\mathrm{Tuj}_{-} \mathrm{1}^{+}$to detect newborn immature neurons and used $\mathrm{BrdU}^{+}-\mathrm{MAP}-2^{+}$to detect newborn mature neurons. Consistent with previous reports ${ }^{[30]}$, we observed that most of these double-staining cells were located at the medial striatum and frontal cortex from the lateral ventricle to the boundary of the ischemic core. Compared with the sham group, BrdU ${ }^{+}-\mathrm{Tuj}-1^{+}$cells and BrdU ${ }^{+}-\mathrm{MAP}-2^{+}$cells increased 

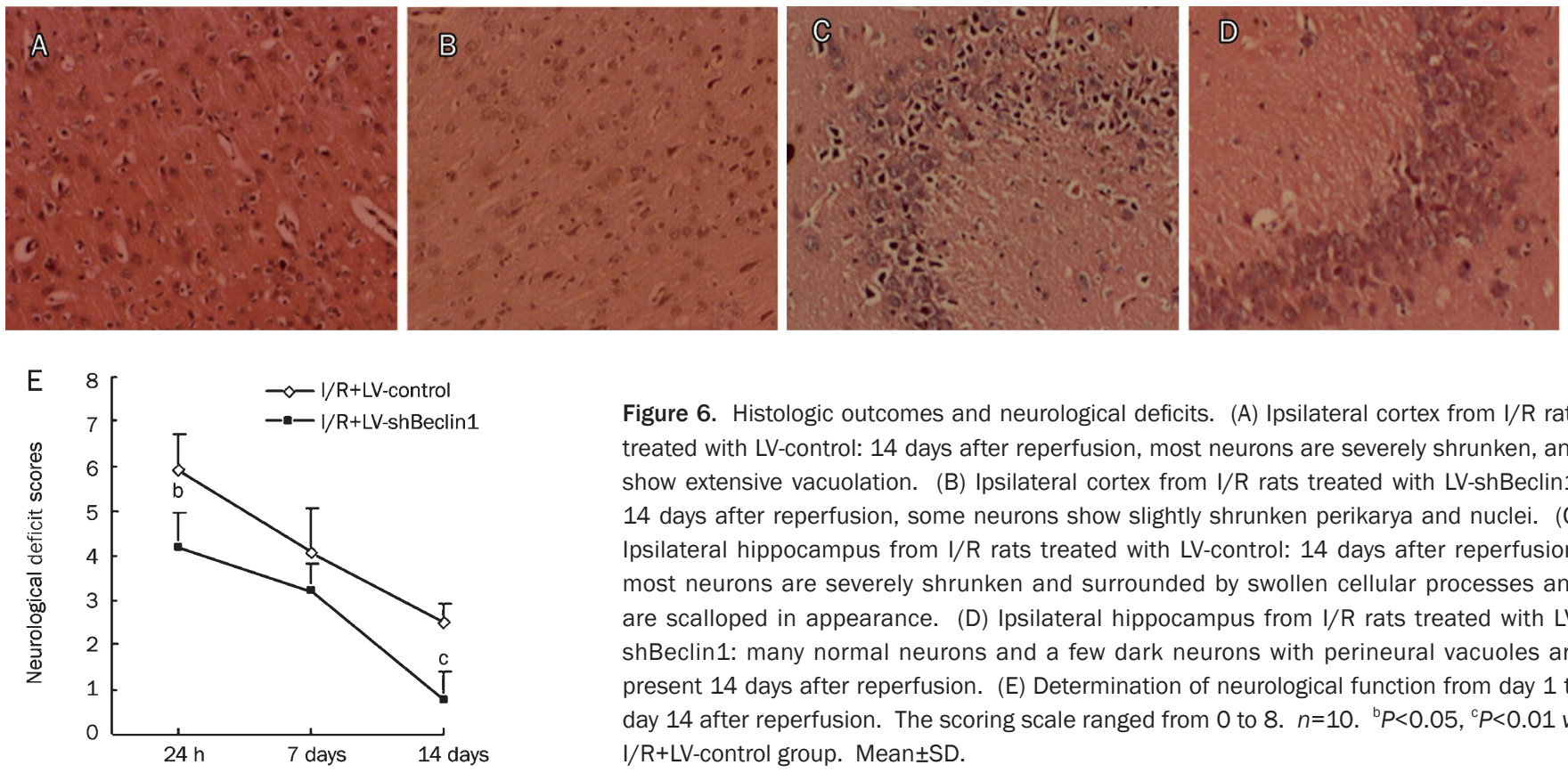

Figure 6. Histologic outcomes and neurological deficits. (A) Ipsilateral cortex from I/R rats treated with LV-control: 14 days after reperfusion, most neurons are severely shrunken, and show extensive vacuolation. (B) Ipsilateral cortex from I/R rats treated with LV-shBeclin1: 14 days after reperfusion, some neurons show slightly shrunken perikarya and nuclei. (C) Ipsilateral hippocampus from I/R rats treated with LV-control: 14 days after reperfusion, most neurons are severely shrunken and surrounded by swollen cellular processes and are scalloped in appearance. (D) Ipsilateral hippocampus from I/R rats treated with LVshBeclin1: many normal neurons and a few dark neurons with perineural vacuoles are present 14 days after reperfusion. (E) Determination of neurological function from day 1 to day 14 after reperfusion. The scoring scale ranged from 0 to $8 . n=10 .{ }^{b} P<0.05,{ }^{c} P<0.01$ vs I/R+LV-control group. Mean \pm SD.
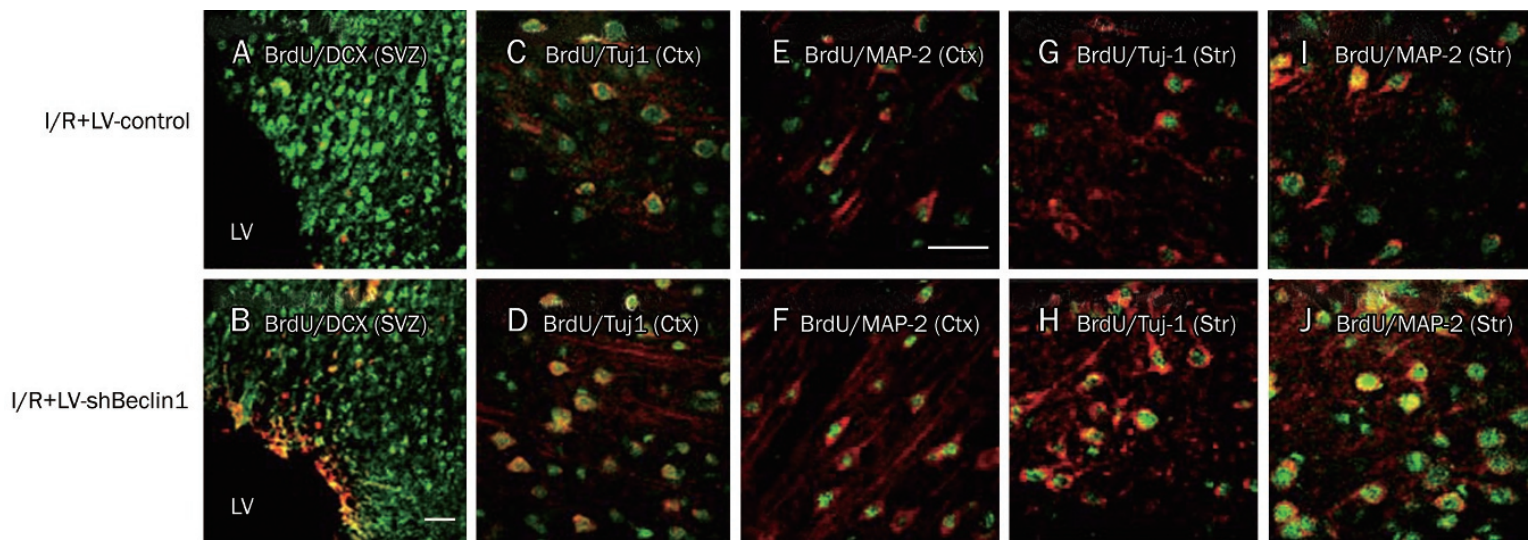

K

SVZ

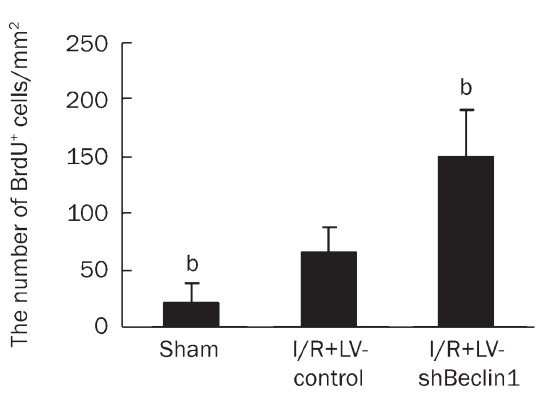

Striatum

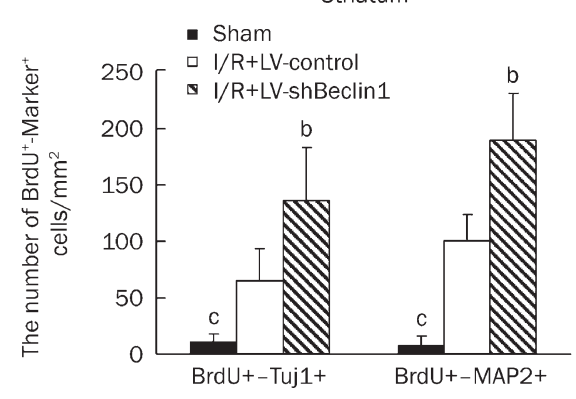

Cortex

- Sham

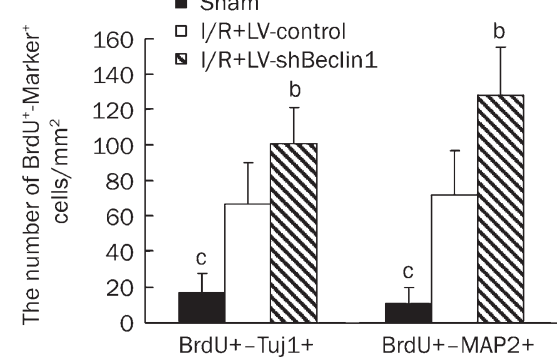

Figure 7. Accumulation, migration and maturity of newborn neurons. (A-B) The merged confocal images confirmed DCX expressing cells (green) and BrdU expressing cells (red) in the SVZ of the ipsilateral hemisphere from I/R animals treated with LV-shBeclin1 or LV-control 14 days after MCAO. (C-F) The merged confocal images show BrdU-labeled cells (green) that were co-labeled with Tuj-1 (red; C-D) and MAP-2 (red; E-F) in the ipsilateral cortex of animals treated with LV-shBeclin1 or LV-control 14 days after MCAO. (G-J): Photomicrographs show BrdU-labeled cells (green) that are co-labeled with Tuj-1 (red; G-H) and MAP-2 (red; I-J) in the ipsilateral striatum from animals treated with LV-shBeclin1 or LV-control 14 days after MCAO. (K) Quantitative data of each cell. An ANOVA test was used for statistical analysis. ${ }^{\mathrm{b}} P<0.05,{ }^{\mathrm{c}} P<0.01 \mathrm{vs} \mathrm{I} / \mathrm{R}+\mathrm{LV}$-control group. $n=6$. Mean \pm SD. Scale bar $=50 \mu \mathrm{m}$ in (B) and scale bar=20 $\mu \mathrm{m}$ in (E). SVZ, subventricular zone; Ctx, Cortex; St, striatum; I/R, Ischemia/Reperfusion. LV, lateral ventricle. 
in the ipsilateral striatum and frontal cortex 14 days postreperfusion. Beclin1 RNAi increased the number of BrdU'Tuj- $1^{+}$cells and BrdU ${ }^{+}-\mathrm{MAP}-2^{+}$cells in the ipsilateral striatum and frontal cortex 14 days after I/R (Figure 7C-J).

\section{Discussion}

RNAi-mediated downregulation of Beclin1 decreases apoptosis and promotes neurogenesis in the ischemic hemisphere, and reduces infarct volume and inhibits histological injury in response to transient MCAO. These findings suggest that Beclin1-dependent autophagy may be a target to repair ischemic injury after stroke.

Autophagy is stimulated by nutrient starvation and growth factor deprivation when cells are unable to take up external nutrients. The process involves the rearrangement of subcellular membranes into autophagosomes and autophagic vacuoles $^{[11,31]}$. Autophagy may be triggered by cerebral ischemia to eliminate damaged mitochondria, thereby aborting apoptosis and delaying necrosis. However, if cerebral reperfusion deficits are not corrected, sustaining a high level of "autophagic stress" will lead to massive lysosomal activation and ultimately cell demise ${ }^{[32]}$. It was reported that hypoxic/ischemic neuronal death was largely prevented by Atg7 deficiency ${ }^{[9,33]}$. Induction of autophagy in the ischemic phase was accompanied by activation of AMP-activated protein kinase (AMPK), whereas ischemia/reperfusion stimulates autophagy through a Beclin1-dependent but AMPK-independent mechanism ${ }^{[5]}$. Here, Beclin1 levels and LC3-II/LC3-I increased together with profuse autophagosomes in the ipsilateral hemisphere in response to MCAO reperfusion (Figure 1,2). The results support the view that the upregulation of Beclin1 is restricted to some neurons and it appears to be a response to an ischemic insult ${ }^{[34]}$.

Apoptosis and autophagy can occur concurrently within damaged neurons, leading to cell death of mixed morphological and biochemical features in stroke $\mathrm{e}^{[6,35]}$. Beclin1 has been proposed to coordinate both apoptosis and autophagy through direct interaction with the anti-apoptotic family members Bcl-2 and/or Bcl-X(L) $)^{[36]}$. The size of myocardial infarction after I/R was significantly smaller in the Beclin1 ${ }^{+/-}$ mice than in wild-type (WT) mice. Furthermore, the number of TUNEL-positive cells in the ischemic area was also smaller in Beclin $1^{+/-}$mice than in WT mice ${ }^{[5]}$. Beclin1-upregulating cells are also the ones that express the active form of caspase$3^{[34]}$, implying that Beclin1 may participate directly in initiating apoptotic signaling. In the present study, we demonstrated that RNAi-mediated downregulation of Beclin1 reduced the apoptosis of immature neurons surrounding the ischemic core (Figure 4). Furthermore, downregulation of Beclin1 by RNAi reduced infarct volume, improved histological outcomes and decreased neurological deficit scores (Figure 5 and 6). These findings indicate that LV-shBeclin1 protects against ischemic brain injury.

Recent studies have demonstrated that neurogenesis from endogenous precursors is induced after focal cerebral ischemia and increasing neurogenesis after stroke could be a therapeu- tic strategy ${ }^{[19,30,37]}$. Modulation of neurodevelopment following brain damage by altering Beclin1-dependent autophagy has been well documented. For example, ethanol promotes premature neural progenitor maturation via suppressing autophagy rather than initiating cell death ${ }^{[21]}$. Ambral is a gene that encodes the activating molecule in Beclin1-regulated autophagy. Mutant Ambral ${ }^{\text {gt/gt }}$ embryos appear to have overgrowth of the nervous system due to impairment of basal autophagy ${ }^{[38]}$. However, it was also reported that Ambra 1, a novel activating molecule in Beclin1-regulated autophagy, plays a role in the development of the nervous system ${ }^{[38]}$. Hence, further investigation is needed to clarify the role of Beclin1-dependent autophagy in neurogenesis after cerebral ischemia. We further studied the effects of stereotaxic injection of LV-shBeclin1 on neurogenesis in the adult rat brain after a transient MCAO. As previously reported ${ }^{[30]}$, we observed that transient MCAO caused the proliferation of neural progenitor cells and promoted the maturation of newborn neurons in the ipsilateral hemisphere. Interestingly, we found that Beclin1 downregulation by RNAi increased the number of neural progenitor cells $\left(\mathrm{BrdU}^{+}-\mathrm{DCX}^{+}\right)$, newborn immature $\left(\mathrm{BrdU}^{+}-\mathrm{Tuj}-1^{+}\right)$ and mature neurons $\left(\mathrm{BrdU}^{+}-\mathrm{MAP}-2^{+}\right)$in the surrounding ischemic core of the ipsilateral hemisphere (Figure 7). These findings support the view that dysregulation of Beclin1-dependent autophagy may be linked to cell proliferation ${ }^{[39,40]}$.

Taken together, the data show that inhibition of Beclin1 by RNAi provides neuroprotection, possibly via promotion of neurogenesis and inhibition of apoptosis. Our findings suggest that Beclin1 downregulation could be a therapeutic strategy to improve recovery after stroke.

\section{Acknowledgments}

This work was supported by Key Project of National Science Foundation of China (№ 30830118) and the International Project of Ministry of Science and Technology (№ 2006DFA31750).

\section{Author contribution}

Prof Jian-xun LIU designed the research; Dr Yong-qiu ZHENG and Dr Xin-zhi LI performed the research and analyzed the data; Prof Li XU and Yong-gang XU contributed new analytical tools and reagents; and Yong-qiu ZHENG wrote the paper.

\section{References}

1 Lee IA, Lee JH, Baek NI, Kim DH. Antihyperlipidemic effect of crocin isolated from the fructus of Gardenia jasminoides and its metabolite Crocetin. Biol Pharm Bull 2005; 28: 2106-10.

2 Zhao BQ, Ikeda Y, Ihara H, Urano T, Fan W, Mikawa S, et al. Essential role of endogenous tissue plasminogen activator through matrix metalloproteinase 9 induction and expression on heparin-produced cerebral hemorrhage after cerebral ischemia in mice. Blood 2004; 103: $2610-6$.

3 Gursoy-Ozdemir Y, Qiu J, Matsuoka N, Bolay H, Bermpohl D, Jin H, et al. Cortical spreading depression activates and upregulates MMP-9. J Clin Invest 2004; 113: 1447-55.

4 Lockshin RA, Zakeri Z. Caspase-independent cell deaths. Curr Opin Cell Biol 2002; 14: 727-33.

5 Matsui Y, Takagi H, Qu X, Abdellatif M, Sakoda H, Asano T, et al. 
Distinct roles of autophagy in the heart during ischemia and reperfusion: roles of AMP-activated protein kinase and Beclin 1 in mediating autophagy. Circ Res 2007; 100: 914-22.

6 Zhu C, Wang X, Xu F, Bahr BA, Shibata M, Uchiyama Y, et al. The influence of age on apoptotic and other mechanisms of cell death after cerebral hypoxia-ischemia. Cell Death Differ 2005; 12: 162-76.

7 Degterev A, Huang Z, Boyce M, Li Y, Jagtap P, Mizushima N, et al. Chemical inhibitor of nonapoptotic cell death with therapeutic potential for ischemic brain injury. Nat Chem Biol 2005; 1: 112-9.

8 Adhami F, Liao G, Morozov YM, Schloemer A, Schmithorst VJ, Lorenz $\mathrm{JN}$, et al. Cerebral ischemia-hypoxia induces intravascular coagulation and autophagy. Am J Pathol 2006; 169: 566-83.

9 Uchiyama Y, Koike M, Shibata M. Autophagic neuron death in neonatal brain ischemia/hypoxia. Autophagy 2008; 4: 404-8.

10 Nitatori T, Sato N, Waguri S, Karasawa Y, Araki H, Shibanai K, et al. Delayed neuronal death in the CA1 pyramidal cell layer of the gerbil hippocampus following transient ischemia is apoptosis. J Neurosci 1995; 15: 1001-11.

11 Klionsky DJ, Emr SD. Autophagy as a regulated pathway of cellular degradation. Science 2000; 290: 1717-21.

12 Meijer AJ, Codogno P. Signalling and autophagy regulation in health, aging and disease. Mol Aspects Med 2006; 27: 411-25.

13 Pattingre S, Tassa A, Qu X, Garuti R, Liang XH, Mizushima N, et al. $\mathrm{Bcl}-2$ antiapoptotic proteins inhibit Beclin 1-dependent autophagy. Cell 2005; 122: 927-39.

14 Wang CW, Klionsky DJ. The molecular mechanism of autophagy. Mol Med 2003; 9: 65-76.

15 Liang XH, Kleeman LK, Jiang HH, Gordon G, Goldman JE, Berry G, et al. Protection against fatal Sindbis virus encephalitis by beclin, a novel Bcl-2-interacting protein. J Virol 1998; 72: 8586-96.

$16 \mathrm{Qu} \mathrm{X,} \mathrm{Yu} \mathrm{J,} \mathrm{Bhagat} \mathrm{G,} \mathrm{Furuya} \mathrm{N,} \mathrm{Hibshoosh} \mathrm{H,} \mathrm{Troxel} \mathrm{A,} \mathrm{et} \mathrm{al.} \mathrm{Promo-}$ tion of tumorigenesis by heterozygous disruption of the beclin 1 autophagy gene. J Clin Invest 2003; 112: 1809-20.

17 Yu L, Alva A, Su H, Dutt P, Freundt E, Welsh S, et al. Regulation of an ATG7-beclin 1 program of autophagic cell death by caspase-8. Science 2004; 304: 1500-2.

18 Boya P, Gonzalez-Polo RA, Casares N, Perfettini JL, Dessen P, Larochette $\mathrm{N}$, et al. Inhibition of macroautophagy triggers apoptosis. Mol Cell Biol 2005; 25: 1025-40.

19 Nygren J, Wieloch T, Pesic J, Brundin P, Deierborg T. Enriched environment attenuates cell genesis in subventricular zone after focal ischemia in mice and decreases migration of newborn cells to the striatum. Stroke 2006; 37: 2824-9.

20 Martinez G, Musumeci G, Loreto C, Carnazza ML. Immunohistochemical changes in vulnerable rat brain regions after reversible global brain ischaemia. J Mol Histol 2007; 38: 295-302.

21 Prock TL, Miranda RC. Embryonic cerebral cortical progenitors are resistant to apoptosis, but increase expression of suicide receptor DISC-complex genes and suppress autophagy following ethanol exposure. Alcohol Clin Exp Res 2007; 31: 694-703.

22 Yue Z, Horton A, Bravin M, DeJager PL, Selimi F, Heintz N. A novel protein complex linking the delta 2 glutamate receptor and autophagy: implications for neurodegeneration in lurcher mice. Neuron 2002; 35: 921-33.

23 Elbashir SM, Harborth J, Weber K, Tuschl T. Analysis of gene function in somatic mammalian cells using small interfering RNAs. Methods
2002; 26: 199-213.

24 Hoehn BD, Palmer TD, Steinberg GK. Neurogenesis in rats after focal cerebral ischemia is enhanced by indomethacin. Stroke 2005; 36 : 2718-24.

25 Bradford MM. A rapid and sensitive method for the quantitation of microgram quantities of protein utilizing the principle of protein-dye binding. Anal Biochem 1976; 72: 248-54.

26 Tsubokawa T, Jadhav V, Solaroglu I, Shiokawa Y, Konishi Y, Zhang JH. Lecithinized superoxide dismutase improves outcomes and attenuates focal cerebral ischemic injury via antiapoptotic mechanisms in rats. Stroke 2007; 38: 1057-62.

27 Xia CF, Smith RS Jr, Shen B, Yang ZR, Borlongan CV, Chao L, et al. Postischemic brain injury is exacerbated in mice lacking the kinin B2 receptor. Hypertension 2006; 47: 752-61.

28 Cuervo AM. Autophagy: many paths to the same end. Mol Cell Biochem 2004; 263: 55-72.

29 Kabeya Y, Mizushima N, Ueno T, Yamamoto A, Kirisako T, Noda T, et al. LC3, a mammalian homologue of yeast Apg8p, is localized in autophagosome membranes after processing. Embo J 2000; 19: 5720-8.

30 Zhang R, Xue YY, Lu SD, Wang Y, Zhang LM, Huang YL, et al. Bcl-2 enhances neurogenesis and inhibits apoptosis of newborn neurons in adult rat brain following a transient middle cerebral artery occlusion. Neurobiol Dis 2006; 24: 345-56.

31 Reggiori F, Klionsky DJ. Autophagosomes: biogenesis from scratch? Curr Opin Cell Biol 2005; 17: 415-22.

32 Chu CT. Autophagic stress in neuronal injury and disease. J Neuropathol Exp Neurol 2006; 65: 423-32.

33 Koike M, Shibata M, Tadakoshi M, Gotoh K, Komatsu M, Waguri S, et al. Inhibition of autophagy prevents hippocampal pyramidal neuron death after hypoxic-ischemic injury. Am J Pathol 2008; 172: 454-69.

34 Rami A, Langhagen A, Steiger S. Focal cerebral ischemia induces upregulation of Beclin 1 and autophagy-like cell death. Neurobiol Dis 2008; 29: 132-41.

35 Yang DS, Kumar A, Stavrides P, Peterson J, Peterhoff CM, Pawlik M, et al. Neuronal apoptosis and autophagy cross talk in aging ps/app mice, a model of Alzheimer's disease. Am J Pathol 2008; 173: 66581.

36 Oberstein A, Jeffrey PD, Shi Y. Crystal structure of the Bcl- $\mathrm{X}_{\mathrm{L}}$-Beclin 1 peptide complex: Beclin 1 is a novel BH3-only protein. J Biol Chem 2007; 282: 13123-32.

37 Sugiura S, Kitagawa K, Tanaka S, Todo K, Omura-Matsuoka E, Sasaki $\mathrm{T}$, et al. Adenovirus-mediated gene transfer of heparin-binding epidermal growth factor-like growth factor enhances neurogenesis and angiogenesis after focal cerebral ischemia in rats. Stroke 2005; 36: 859-64.

38 Cecconi F, Di Bartolomeo S, Nardacci R, Fuoco C, Corazzari M, Giunta $\mathrm{L}$, et al. A novel role for autophagy in neurodevelopment. Autophagy 2007; 3: 506-8.

39 Fimia GM, Stoykova A, Romagnoli A, Giunta L, Di Bartolomeo S, Nardacci R, et al. Ambra1 regulates autophagy and development of the nervous system. Nature 2007; 447: 1121-5.

40 Kuida K, Haydar TF, Kuan CY, Gu Y, Taya C, Karasuyama H, et al. Reduced apoptosis and cytochrome $c$-mediated caspase activation in mice lacking caspase 9. Cell 1998; 94: 325-37. 